\title{
A class of Caffarelli-Kohn-Nirenberg type inequalities on the H-type group ${ }^{1}$
}

\author{
Shutao Zhang(*) - YaZhou Han(*) - JingBo Dou(**)
}

ABstRact - This work is devoted to establish a class of Caffarelli-Kohn-Nirenberg type inequalities on the H-type group. Inspired by the idea of Chern J.L. and Lin C.S., a function transformation is introduced. Combining some elementary inequalities and some accurate estimates, we establish a class of weighted Hardy-Sobolev type inequalities and then obtain our main result, namely Caffarelli-Kohn-Nirenberg type inequalities on the H-type group.

Mathematics Subject Classification (2010). 35H20; 26D10; $22 \mathrm{E} 30$.

KeYwords. Caffarelli-Kohn-Nirenberg type inequality; Hardy-Sobolev type inequality; H-type group.

\section{Introduction}

In [4], Caffarelli, Kohn, and Nirenberg established the following first order interpolation inequalities with weights, known as Caffarelli-KohnNirenberg (CKN) inequalities.

$\left({ }^{1}\right)$ Supported by the National Natural Science Foundation of China (Grant No. 11201443 and No. 11101319) and Zhejiang Provincial Natural Science Foundation of China (Grant No. Y6110118).

(*) Indirizzo degli A.: Department of Mathematics, China Jiliang University, Hangzhou 310018, P.R. China.

E-mail: taoer558@163.com yazhou.han@gmail.com

(**) Indirizzo dell'A.: School of Statistics, Xi'an University of Finance and Economics, Xi'an, 710100, P.R. China.

E-mail:djbmn@126.com 
Theorem A Let $p, q, r, \alpha, \beta, \gamma, \sigma$ and a satisfy

$$
\left\{\begin{array}{l}
p, q \geq 1, r>0,0 \leq a \leq 1 \\
\frac{1}{p}+\frac{\alpha}{n}, \frac{1}{q}+\frac{\beta}{n}, \frac{1}{r}+\frac{\gamma}{n}>0,
\end{array}\right.
$$

where $\gamma=a \sigma+(1-a) \beta$. Then there exists a positive constant $C$ such that the following inequality holds for all $u \in C_{0}^{\infty}\left(R^{n}\right)$

$$
\left\|\left.\left.x\right|^{\gamma} u\right|_{L^{r}} \leq\left.\left. C|| x\right|^{\alpha}\left|\nabla u \|_{L^{p}}^{a}\right||x|^{\beta} u\right|_{L^{q}} ^{1-a}\right.
$$

if and only if the following relations hold:

$$
\frac{1}{r}+\frac{\gamma}{n}=a\left(\frac{1}{p}+\frac{\alpha-1}{n}\right)+(1-a)\left(\frac{1}{q}+\frac{\beta}{n}\right)
$$

(this is dimensional balance),

$$
\begin{aligned}
& 0 \leq \alpha-\sigma \quad \text { if } a>0 \\
& \alpha-\sigma \leq 1 \quad \text { if } \alpha>0 \text { and } \quad \frac{1}{p}+\frac{\alpha-1}{n}=\frac{1}{r}+\frac{\gamma}{n} .
\end{aligned}
$$

Furthermore, on any compact set in parameter space in which (1.1), (1.3) and $0 \leq \alpha-\sigma \leq 1$ hold, the constant $C$ is bounded.

It is well known that $\mathrm{CKN}$ inequalities include some important integral inequalities. For instance, the case $a=1, \gamma=\alpha-1$ and the case $a=1, \alpha=\gamma=0$ are corresponding to Hardy inequality[9] and Sobolev inequality, respectively. While the case $a=1$ is Hardy-Sobolev inequality[1]. So, CKN inequalities can be expected to apply more extensively to related $\mathrm{PDEs}$.

In 1986, Theorem A was extended to the higher order case by Lin C.S. (see [21]). In [13], Gutierrez and Wheeden gave a class of weighted local Sobolev interpolation inequalities and derived global Laudau inequalities. For applications, many authors have discussed the best constants and extremal functions of CKN inequalities (see e.g. [5],[20]), which play important roles in many problems, such as eigenvalue problems, existence problems of equation with singular weights, regularity problems, etc. (see [1], [3], [4], [5], [6], [7], [9], [12], [13], [20], [21] and references therein).

H-type group $G$ was introduced by Kaplan in [18], which is a class of Carnot group. If the dimension of the center of Lie algebra of an H-type group is trivial, then the H-type group is isomorphic to Heisenberg group. Analysis on the H-type group is motivated by its role as an important 
model in the general theory of vector fields satisfying Hörmander's condition (see [2], [11], [10], [14], [15], [17], [18], [24]).

Some important integral inequalities have been obtained and applied to related problems. For example, Hardy-Sobolev type inequality has been obtained by the second author and Niu ([15]) On the H-type group. Therefore, we desire to know whether CKN inequality in Theorem $\mathbf{A}$ is true on the H-type group. This paper will give a positive answer for the case $1<p<Q$, where $Q$ is homogeneous dimension of H-type group.

To state our results, we need to describe some preliminaries for $\mathrm{H}$-type group $G$ (more details can be found in [10], [11], [18] and references therein).

Consider a Carnot group $G$ of step 2, with Lie algebra $\mathbf{g}=V_{1} \oplus V_{2}$. We assume that $\mathbf{g}$ is equipped with a scalar product $\langle\cdot, \cdot\rangle$, with respect to which the $V_{j}^{\prime}$ s are mutually orthogonal. We use the exponential mapping $\exp : \mathbf{g} \rightarrow G$ to define analytic maps $\xi_{i}: G \rightarrow V_{i}, i=1,2$, through the equation $g=\exp \left(\xi_{1}(g)+\xi_{2}(g)\right)$. Here, $\xi(g)=\xi_{1}(g)+\xi_{2}(g)$ is such that $g=\exp (\xi(g))$. With $m=\operatorname{dim}\left(V_{1}\right)$ and $V_{1}=\operatorname{span}\left\{X_{1}, \cdots, X_{m}\right\}\left(X_{1}, \cdots, X_{m}\right.$ are orthonormal), the coordinates of the projection $\xi_{1}$ in the basis $X_{1}, \cdots, X_{m}$ are denoted by $x_{1}=x_{1}(g), \cdots, x_{m}=x_{m}(g)$; that is,

$$
x_{j}(g)=\left\langle\xi(g), X_{j}\right\rangle, \quad j=1, \cdots, m,
$$

and we set $x=x(g)=\left(x_{1}(g), \cdots, x_{m}(g)\right) \in R^{m}$. Similarly, for a fixed orthonormal basis $Y_{1}, \cdots, Y_{k}$ of $V_{2}$, we define the exponential coordinates in the second layer $V_{2}$ of a point $g \in G$ with

$$
y_{i}(g)=\left\langle\xi(g), Y_{i}\right\rangle, \quad i=1, \cdots, k
$$

and $y=\left(y_{1}, \cdots, y_{k}\right) \in R^{k}$.

For each $v \in V_{1}$, consider the orthogonal decomposition

$$
V_{1}=K_{v} \oplus R_{v},
$$

where $K_{v}=\operatorname{ker}\left(a d_{v}: V_{1} \rightarrow V_{2}\right)=\left\{v^{\prime} \in V_{1}:\left[v, v^{\prime}\right]=0\right\}$. We say that the Lie algebra $\mathbf{g}$ is of H-type if the mapping $a d_{v}: R_{v} \rightarrow V_{2}$ is a surjective isometry for every unit vector $v \in V_{1}$ and the corresponding simple connected group $G$ is named H-type group.

A family of dilations is defined by

$$
\delta_{\lambda}(x, y)=\left(\lambda x, \lambda^{2} y\right), \quad \text { for any } \lambda>0,(x, y) \in G .
$$

The homogeneous dimension of $G$ with respect to dilations is $Q=m+2 k$.

Recently, using Hardy-Sobolev type inequality and interpolation technique in [17], a class of CKN type inequalities on the H-type group has been obtained as follows: 
Theorem B Set $p, q, r, \beta, \gamma, \sigma$ and a such that

$$
\left\{\begin{array}{l}
1<p<Q, \quad q, r \geq 1, \quad 0 \leq a \leq 1, \\
m>\gamma r>-Q, \quad m>\beta q>-Q,
\end{array}\right.
$$

where

$$
\gamma=a \sigma+(1-a) \beta
$$

For any $u \in C_{0}^{\infty}(G)$, there exists a positive constant $C$ such that

$$
\begin{aligned}
& \left(\int_{G} \frac{|x|^{-\gamma r}}{\rho^{-\gamma r}} \rho^{\gamma r}|u|^{r} d x d y\right)^{\frac{1}{r}} \\
\leq & C\left(\int_{G}|X u|^{p} d x d y\right)^{\frac{a}{p}}\left(\int_{G} \frac{|x|^{-\beta q}}{\rho^{-\beta q}} \rho^{\beta q}|u|^{q} d x d y\right)^{\frac{1-a}{q}}
\end{aligned}
$$

holds if and only if the following relations hold:

$$
\frac{1}{r}+\frac{\gamma}{Q}=a\left(\frac{1}{p}-\frac{1}{Q}\right)+(1-a)\left(\frac{1}{q}+\frac{\beta}{Q}\right)
$$

((1.7) is dimensional balance),

$$
\begin{aligned}
& 0 \geq \sigma, \quad \text { if } a>0, \\
& \sigma \geq-1, \quad \text { if } a>0 \text { and } \frac{1}{p}-\frac{1}{Q}=\frac{1}{r}+\frac{\gamma}{Q},
\end{aligned}
$$

where $X u=\left(X_{1} u, \cdots, X_{m} u\right)$ is the generalized horizontal gradient, $\rho=\left(|x(g)|^{4}+\left.|16| y(g)\right|^{2}\right)^{\frac{1}{4}}$ is the norm function and dxdy is the Haar measure on $G$.

Obviously, Theorem $\mathbf{B}$ had initially investigated that the CKN inequality holds in the specific model of vector fields satisfying Hörmander's condition. However, it is not completely analogous to Theorem A.

In this paper, we will establish the following generalizing CKN type inequalities on the H-type group $G$.

Theorem 1.1. Set $p, q, r, \alpha, \beta, \gamma, \sigma$ and $a$ such that

$$
\left\{\begin{array}{l}
1<p<Q, \quad q \geq 1, \quad r>0, \quad 0 \leq a \leq 1, \\
\gamma r+Q>0, \quad \alpha p+Q>0, \quad \beta q+Q>0, \\
m+(\alpha-\gamma) r>0, \quad m+(\alpha-\beta) q>0,
\end{array}\right.
$$


where

$$
\gamma=a \sigma+(1-a) \beta
$$

For any $u \in C_{0}^{\infty}(G)$, there exists a positive constant $C$ such that

$$
\begin{aligned}
& \left(\int_{G}\left(\frac{|x|}{\rho}\right)^{(\alpha-\gamma) r} \rho^{\gamma r}|u|^{r} d x d y\right)^{\frac{1}{r}} \\
\leq & C\left(\int_{G}\left|\rho^{\alpha} X u\right|^{p} d x d y\right)^{\frac{a}{p}}\left(\int_{G}\left(\frac{|x|}{\rho}\right)^{(\alpha-\beta) q} \rho^{\beta q}|u|^{q} d x d y\right)^{\frac{1-a}{q}}
\end{aligned}
$$

holds if and only if the following relations hold:

$$
\frac{1}{r}+\frac{\gamma}{Q}=a\left(\frac{1}{p}+\frac{\alpha-1}{Q}\right)+(1-a)\left(\frac{1}{q}+\frac{\beta}{Q}\right)
$$

(the dimensional balance condition),

$$
\begin{aligned}
& \alpha-\sigma \geq 0, \quad \text { if } a>0, \\
& \alpha-\sigma \leq 1, \quad \text { if } a>0 \text { and } \frac{1}{p}+\frac{\alpha-1}{Q}=\frac{1}{r}+\frac{\gamma}{Q} .
\end{aligned}
$$

To prove Theorem 1.1, we employ the idea of [16], [17]. So we need firstly to establish a class of weighted Hardy-Sobolev type inequalities as follows:

Theorem 1.2. If $1<p<Q, 0 \leq s \leq p, \alpha>\frac{p-Q}{p}, p_{*}(s, p, Q)=\frac{p(Q-s)}{Q-p}$, then there exists a positive constant $C=C(s, p, \alpha, Q)$ such that for every $u \in D_{\alpha}^{1, p}\left(H_{n}\right)$,

$$
\int_{G} \frac{|x|^{s} \mid}{\rho^{s}} \frac{\left|\rho^{\alpha} u\right|^{p_{*}(s, p, Q)}}{\rho^{s}} d x d y \leq C\left(\int_{G}\left|\rho^{\alpha} X u\right|^{p} d x d y\right)^{\frac{Q-s}{Q-p}},
$$

where $D_{\alpha}^{1, p}(G)$ is the closure of $C_{0}^{\infty}(G)$ with respect to the norm

$$
\|u\|_{\alpha}^{p}=\int_{G}\left|\rho^{\alpha} X u\right|^{p} d x d y .
$$

Remark 1.3. When $\alpha=0$, Theorem 1.2 and Theorem 1.1 are HardySobolev type inequality (see [15]) and CKN type inequality (see [17]), re- 
spectively. When $a=1$, conditions of Theorem 1.1 imply

$$
0 \leq \alpha-\sigma=\alpha-\gamma \leq 1, \quad \frac{1}{r}+\frac{\sigma}{Q}=\frac{1}{p}+\frac{\alpha-1}{Q},
$$

and then $p \leq r \leq p^{*}=\frac{Q p}{Q-p}$. So, there exists $t \in[0,1]$ such that

$$
r=t p+(1-t) p^{*}=\frac{p(Q-t p)}{Q-p},
$$

and $(\alpha-\sigma) r=t p$. Substituting $r$ and $\alpha-\sigma$, (1.12) becomes the weighted Hardy-Sobolev type inequality (1.16).

As far as we know, on the H-type group, there are fewer inequalities, which are similar to (1.6) and (1.12). Main known results were derived by $\mathrm{Lu}$ in [22], [23] on stratified nilpotent Lie group. There, through approximating Sobolev functions by polynomials, Lu gave both local and global Sobolev interpolation inequalities (weighted and nonweighted version) of any higher orders for the Folland-Stein Sobolev spaces on stratified nilpotent Lie group and on Boman chain domains. But, these results are different from the results of Theorem B and Theorem 1.1.

The paper is organized as follows. In Section 2, we introduce some basic facts for the H-type group. Section 3 is devoted to the proof of Theorem 1.2. In Section 4, we complete the proof of our main result, namely Theorem 1.1, by using some accurate estimates and weighted Hardy-Sobolev type inequality.

\section{Some known results}

In [10], [11], Garofalo and Vassilev pointed out that

$$
\begin{aligned}
X_{j} & =\frac{\partial}{\partial x_{j}}+\frac{1}{2} \sum_{i=1}^{k}\left\langle\left[\xi, X_{j}\right], Y_{i}\right\rangle \frac{\partial}{\partial y_{i}} \\
& =\frac{\partial}{\partial x_{j}}+\frac{1}{2} \sum_{i=1}^{k}\left\langle\left[\xi_{1}, X_{j}\right], Y_{i}\right\rangle \frac{\partial}{\partial y_{i}}, j=1, \ldots, m,
\end{aligned}
$$

where $\xi=\xi_{1}+\xi_{2} \in \mathbf{g}=V_{1} \oplus V_{2}, x=\left(x_{1}, \ldots, x_{m}\right) \in R^{m}, y=\left(y_{1}, \ldots, y_{k}\right) \in R^{k}$. Denote by

$$
\begin{aligned}
& X u=\left(X_{1} u, X_{2} u, \ldots, X_{m} u\right), \\
& \operatorname{div}_{X}\left(u_{1}, u_{2}, \cdots, u_{m}\right)=\sum_{i=1}^{m} X_{i} u_{i},
\end{aligned}
$$


and

$$
L u=\sum_{i=1}^{m} X_{i}^{2} u
$$

the horizontal gradient, horizontal divergence and sub-Laplacian, respectively.

The norm function on $G$ has the form

$$
\rho(g)=d(g, 0)=\left(|x(g)|^{4}+16|y(g)|^{2}\right)^{\frac{1}{4}},
$$

where 0 is the unit element of $G$. It is easy to verify that (see [10], [11])

$$
\begin{aligned}
& \left|X\left(|x(g)|^{2}\right)\right|^{2}=4|x(g)|^{2}, \quad\left|X\left(|y(g)|^{2}\right)\right|^{2}=|x(g)|^{2}|y(g)|^{2}, \\
& \left\langle X\left(|x(g)|^{2}\right), X\left(|y(g)|^{2}\right)\right\rangle=0, \quad|X \rho|^{2}=\frac{|x(g)|^{2}}{\rho^{2}}, \\
& L\left(|x(g)|^{2}\right)=2 m, \quad L\left(|y(g)|^{2}\right)=\frac{k}{2}|x(g)|^{2}, \\
& L \rho=(Q-1) \frac{|x(g)|^{2}}{\rho^{3}}, \quad\langle X(|x(g)|), X \rho\rangle=\frac{|x(g)|^{3}}{\rho^{3}} .
\end{aligned}
$$

Denote by $B_{R}(\xi) \equiv B(\xi, R)=\left\{\eta \in G \mid d(\xi, \eta)=\rho\left(\eta^{-1} \xi\right)<R\right\}$ the ball centered at $\xi$ with radius $R$ and let $\left(x^{*}, y^{*}\right)=\left(\frac{x}{\rho}, \frac{y}{\rho^{2}}\right)$ be a point on the unit sphere $S=\{g \in G: \rho(g)=1\}$.

The polar coordinates formula (see [2], [8], [14]) is stated as follows: There is a unique Radon measure $d \mu$ on $S$ such that for all $f \in L^{1}(G)$,

$$
\int_{G} f(x, y) d x d y=\int_{0}^{\infty} \int_{S} f \circ \delta_{\rho}\left(x^{*}, y^{*}\right) \rho^{Q-1} d \mu d \rho .
$$

Moreover, authors of [2], [14] gave the explicit polar coordinates transformation, Jacobian and the following criteria for the integrability of $|x|^{p} \rho^{q}$.

1) If $p+m>0$ and $p+q+Q>0$, then $\int_{B_{1}}|x|^{p} \rho^{q} d x d y<+\infty$;

2) If $p+m>0$ and $p+q+Q<0$, then $\int_{G \backslash \overline{B_{1}}}|x|^{p} \rho^{q} d x d y<+\infty$. Setting

$$
A=\left(\int_{G}\left|\rho^{\alpha} X u\right|^{p} d x d y\right)^{\frac{1}{p}}, \quad B=\left(\int_{G} \frac{|x|^{(\alpha-\beta) q}}{\rho^{(\alpha-\beta) q}} \rho^{\beta q}|u|^{q} d x d y\right)^{\frac{1}{q}},
$$


(1.12) can be rewritten as

$$
\left(\int_{G} \frac{|x|^{(\alpha-\gamma) r}}{\rho^{(\alpha-\gamma) r}} \rho^{\gamma r}|u|^{r} d x d y\right)^{\frac{1}{r}} \leq C A^{a} B^{1-a} .
$$

Rescaling $u$ such that $A^{a} B^{1-a}=1$, our goal becomes to investigate the following inequality

$$
\left(\int_{G} \frac{|x|^{(\alpha-\gamma) r}}{\rho^{(\alpha-\gamma) r}} \rho^{\gamma r}|u|^{r} d x d y\right)^{\frac{1}{r}} \leq C .
$$

In the sequel, let $\Phi(\xi)(0 \leq \Phi \leq 1)$ be a fixed $C_{0}^{\infty}$ function on $G$ satisfying

$$
\Phi= \begin{cases}1, & \text { if } \rho<\frac{1}{2} \\ 0, & \text { if } \rho>1\end{cases}
$$

\section{Weighted Hardy-Sobolev type inequality}

In this section, we devote to establish a weighted Hardy-Sobolev type inequality. To this end, we introduce the following known facts firstly.

Proposition 3.1 [Theorem 4.2, [17]]. Let $p \geq 1, \alpha \in R$ and $\frac{1}{p}+$ $\frac{\alpha-1}{Q}>0$. For any $f \in C_{0}^{\infty}(G)$, there exists a positive constant $C=\left(\frac{p}{Q-p+\alpha p}\right)^{p}$ such that

$$
\int_{G} \rho^{\alpha p} \frac{|x|^{p}}{\rho^{p}} \frac{|f|^{p}}{\rho^{p}} d x d y \leq C \int_{G} \rho^{\alpha p}|X f|^{p} d x d y
$$

Proposition 3.2 [see [3]]. For all $\xi_{1}, \xi_{2} \in R^{n}$, the following inequalities hold:

1). If $p \leq 2$,

$$
\begin{gathered}
\left|\xi_{1}+\xi_{2}\right|^{p}-\left|\xi_{1}\right|^{p}-p\left|\xi_{1}\right|^{p-2}\left\langle\xi_{1}, \xi_{2}\right\rangle \leq C(p)\left|\xi_{2}\right|^{p} \\
\left|\xi_{2}\right|^{p}-\left|\xi_{1}\right|^{p}-p\left|\xi_{1}\right|^{p-2}\left\langle\xi_{1}, \xi_{2}-\xi_{1}\right\rangle \geq C(p) \frac{\left|\xi_{2}-\xi_{1}\right|^{p}}{\left(\left|\xi_{1}\right|+\left|\xi_{2}\right|\right)^{2-p}}
\end{gathered}
$$


2). If $p>2$,

$$
\begin{gathered}
\left|\xi_{1}+\xi_{2}\right|^{p}-\left|\xi_{1}\right|^{p}-p\left|\xi_{1}\right|^{p-2}\left\langle\xi_{1}, \xi_{2}\right\rangle \leq \frac{p(p-1)}{2}\left(\left|\xi_{1}\right|+\left.\left|\xi_{2}\right|\right|^{p-2}\left|\xi_{2}\right|^{2},\right. \\
\left|\xi_{2}\right|^{p}-\left|\xi_{1}\right|^{p}-p\left|\xi_{1}\right|^{p-2}\left\langle\xi_{1}, \xi_{2}-\xi_{1}\right\rangle \geq C(p)\left|\xi_{2}-\xi_{1}\right|^{p},
\end{gathered}
$$

where $\left\langle\xi_{1}, \xi_{2}\right\rangle$ represents the common inner product.

Proposition 3.3 (Lemma 2.1, [19]). For any $\xi, \eta \in R^{n}$ and $\lambda>0$,

$$
|\xi|^{\lambda+1}+\lambda|\eta|^{\lambda+1}-(\lambda+1)|\eta|^{\lambda-1}\langle\xi, \eta\rangle \geq 0 .
$$

Moreover, the equality holds if and only if $\xi=\eta$.

Proof of Theorem 1.2: The condition $\alpha>\frac{p-Q}{p}$ deduces that

$$
\alpha p_{*}(s, p, Q)-s+Q>0, p \alpha+Q>0,
$$

which ensure that the right and left integral of (1.16) are well defined. For any $u \in C_{0}^{\infty}(G)$, take $w=\rho^{\alpha} u$ and then

$$
\begin{aligned}
\int_{G}|X w|^{p} d x d y & =\int_{G}\left|\rho^{\alpha} X u+\alpha \rho^{\alpha-1} u X \rho\right|^{p} d x d y \\
& \leq \int_{G}\left(\rho^{\alpha}|X u|+|\alpha| \rho^{\alpha-1}|u| \frac{|x|}{\rho}\right)^{p} d x d y \\
& \leq C \int_{G}\left(\rho^{p \alpha}|X u|^{p}+|\alpha|^{p}\left|\rho^{\alpha-1} u\right|^{p}\left(\frac{|x|}{\rho}\right)^{p}\right) d x d y .
\end{aligned}
$$

From (3.1), we see that $\int_{G}|X w|^{p} d x d y<+\infty$, i.e., $w \in D_{0}^{1, p}(G)$. A straightforward computation shows that

$$
\int_{G} \frac{|x|^{s}}{\rho^{s}} \frac{\left|\rho^{\alpha} u\right|^{p_{*}(s, p, Q)}}{\rho^{s}} d x d y=\int_{G} \frac{|x|^{s}}{\rho^{s}} \frac{|w|^{p_{*}(s, p, Q)}}{\rho^{s}} d x d y
$$

and

$$
\int_{G}\left|\rho^{\alpha} X u\right|^{p} d x d y=\int_{G}\left|X w-\alpha \rho^{-1} w X \rho\right|^{p} d x d y .
$$

According to the Proposition 3.2, we will discuss under the case $p \leq 2$ and $p>2$, respectively. 
$3.1-$ Case $p \leq 2$

By the first case of Proposition 3.2, it holds

$$
\begin{aligned}
& C(p) \int_{G}\left|X w-\alpha \rho^{-1} w X \rho\right|^{p} d x d y \\
& \geq \int_{G}\left\{|X w|^{p}-|\alpha|^{p} \frac{|w|^{p}}{\rho^{p}}|X \rho|^{p}\right. \\
& \left.\quad-p \alpha|\alpha|^{p-2} \frac{w|w|^{p-2}}{\rho^{p-1}}|X \rho|^{p-2}\left\langle X \rho, X w-\alpha \rho^{-1} w X \rho\right\rangle\right\} d x d y \\
& =\int_{G}\left\{|X w|^{p}+(p-1)|\alpha|^{p} \frac{|x|^{p}}{\rho^{p}} \frac{|w|^{p}}{\rho^{p}}\right. \\
& \left.\quad-p \alpha|\alpha|^{p-2} \frac{w|w|^{p-2}}{\rho^{p-1}}|X \rho|^{p-2}\langle X \rho, X w\rangle\right\} d x d y .
\end{aligned}
$$

Since

$$
\begin{aligned}
& p \alpha|\alpha|^{p-2} \int_{G} \frac{w|w|^{p-2}}{\rho^{p-1}}|X \rho|^{p-2}\langle X \rho, X w\rangle d x d y \\
= & \alpha|\alpha|^{p-2} \int_{G} \rho^{1-p}|X \rho|^{p-2}\left\langle X \rho, X|w|^{p}\right\rangle d x d y \\
= & -\alpha|\alpha|^{p-2} \int_{G}|w|^{p} \operatorname{div}_{X}\left(\rho^{1-p}|X \rho|^{p-2} X \rho\right) d x d y \\
= & -\alpha|\alpha|^{p-2}(Q-p) \int_{G} \frac{|x|^{p}}{\rho^{p}} \frac{|w|^{p}}{\rho^{p}} d x d y,
\end{aligned}
$$

it follows

$$
\begin{aligned}
C(p) \int_{G} \mid X w- & \left.\alpha \rho^{-1} w X \rho\right|^{p} d x d y \geq \int_{G}|X w|^{p} d x d y \\
& +\alpha|\alpha|^{p-2}(Q-p+\alpha(p-1)) \int_{G} \frac{|x|^{p}}{\rho^{p}} \frac{|w|^{p}}{|x|^{p}} d x d y .
\end{aligned}
$$

Because of $\alpha>\frac{p-Q}{p}$, it knows that $Q-p+\alpha(p-1)>0$. If $\alpha<0$, com- 
bining the Hardy type inequality (3.1), we can deduce from (3.3) and (3.4) that

$$
C(p) \int_{G}\left|\rho^{\alpha} X u\right|^{p} d x d y \geq C_{1} \int_{G}|X w|^{p} d x d y
$$

where

$$
\begin{aligned}
C_{1} & =1+\alpha|\alpha|^{p-2}(Q-p+\alpha(p-1))\left(\frac{p}{Q-p}\right)^{p} \\
& =\left(\frac{p}{Q-p}\right)^{p}\left\{\left(\frac{Q-p}{p}\right)^{p}+\alpha|\alpha|^{p-2}(Q-p)+|\alpha|^{p}(p-1)\right\} .
\end{aligned}
$$

Choosing $\xi=\frac{p-Q}{p}, \eta=\alpha$ in Proposition 3.3, we have $C_{1}>0$. If $\alpha \geq 0$, (3.5) holds obviously with $C_{1}=1$. Then, (1.16) can be obtained by Hardy-Sobolev type inequality (see [15], namely the case $\alpha=0$ in (1.16)).

$3.2-$ Case $p>2$

Taking $\xi_{1}=\alpha \rho^{-1} w X \rho, \xi_{2}=X w-\alpha \rho^{-1} w X \rho$ in Proposition 3.2,

$$
\begin{aligned}
& 2^{p-2} \frac{p(p-1)}{2}\left(\left|\alpha \rho^{-1} w X \rho\right|+|X w|\right)^{p-2}\left|X w-\alpha \rho^{-1} w X \rho\right|^{2} \\
& \geq \frac{p(p-1)}{2}\left(2\left|\alpha \rho^{-1} w X \rho\right|+|X w|\right)^{p-2}\left|X w-\alpha \rho^{-1} w X \rho\right|^{2} \\
& \geq \frac{p(p-1)}{2}\left(\left|\alpha \rho^{-1} w X \rho\right|+\left|X w-\alpha \rho^{-1} w X \rho\right|\right)^{p-2} \\
& \quad \cdot\left|X w-\alpha \rho^{-1} w X \rho\right|^{2}|X w|^{p}-\left|\alpha \rho^{-1} w X \rho\right|^{p} \\
& \quad-p\left|\alpha \rho^{-1} w X \rho\right|^{p-2}\left\langle\alpha \rho^{-1} w X \rho, X w-\alpha \rho^{-1} w X \rho\right\rangle \\
&=|X w|^{p}+(p-1)|\alpha|^{p} \frac{|x|^{p}}{\rho^{p}} \frac{|w|^{p}}{\rho^{p}} \\
& \quad-p \alpha|\alpha|^{p-2}|X \rho|^{p-2}\left\langle\rho^{1-p} X \rho, w|w|^{p-2} X w\right\rangle .
\end{aligned}
$$

Denote by $C_{1}=2^{p-2} \frac{p(p-1)}{2}$ and then 


$$
\begin{aligned}
& C_{1} \int_{G}\left(\left|\alpha \rho^{-1} w X \rho\right|+|X w|\right)^{p-2}\left|X w-\alpha \rho^{-1} w X \rho\right|^{2} d x d y \\
\geq & \int_{G}\left\{|X w|^{p}+(p-1)|\alpha|^{p} \frac{|x|^{p}}{\rho^{p}} \frac{|w|^{p}}{\rho^{p}}\right. \\
& \left.\quad-p \alpha|\alpha|^{p-2}|X \rho|^{p-2}\left\langle\rho^{1-p} X \rho, w|w|^{p-2} X w\right\rangle\right\} d x d y \\
= & \int_{G}\left\{|X w|^{p}+\alpha|\alpha|^{p-2}(Q-p+\alpha(p-1)) \frac{|x|^{p}}{\rho^{p}} \frac{|w|^{p}}{\rho^{p}}\right\} d x d y \\
\geq & C_{2} \int_{G}|X w|^{p} d x d y,
\end{aligned}
$$

where $C_{2}>0$ and the argument of the last inequality in (3.6) is similar to the case $p \leq 2$.

On the other hand, by Hölder inequality, Minkowski inequality and Hardy type inequality (3.1), it is obtained that

$$
\begin{aligned}
& C_{1} \int_{G}\left(\left|\alpha \rho^{-1} w X \rho\right|+|X w|\right)^{p-2}\left|X w-\alpha \rho^{-1} w X \rho\right|^{2} d x d y \\
& \leq C_{1}\left(\int_{G}\left|X w-\alpha \rho^{-1} w X \rho\right|^{p} d x d y\right)^{\frac{2}{p}} \\
& \left(\int_{G}\left(\left|\alpha \rho^{-1} w X \rho\right|+|X w|\right)^{p} d x d y\right)^{\frac{p-2}{p}} \\
& \leq C_{1}\left(\int_{G}\left|X w-\alpha \rho^{-1} w X \rho\right|^{p} d x d y\right)^{\frac{2}{p}} \\
& \left\{\left(\int_{G}\left|\alpha \rho^{-1} w X \rho\right|^{p} d x d y\right)^{\frac{1}{p}}+\left(\int_{G}|X w|^{p} d x d y\right)^{\frac{1}{p}}\right\}^{p-2} \\
& \leq C_{1}\left(1+|\alpha| \frac{p}{Q-p}\right)^{p-2}\left(\int_{G}|X w|^{p} d x d y\right)^{\frac{p-2}{p}} \\
& \left(\int_{G}\left|X w-\alpha \rho^{-1} w X \rho\right|^{p} d x d y\right)^{\frac{2}{p}} \text {. }
\end{aligned}
$$


Combining (3.6) and (3.7), there exists a constant $C_{3}>0$ such that

$$
C_{3} \int_{G}|X w|^{p} d x d y \leq \int_{G}\left|X w-\alpha \rho^{-1} w X \rho\right|^{p} d x d y .
$$

From the Hardy-Sobolev type inequality on the H-type group, it arrives at (1.16).

\section{CKN type inequalities with $1<p<Q$}

For the necessity of Theorem 1.1, since the detailed computations can be found in [17], [16], [4], we give only a simple sketch as follows: (1.13) and (1.14) can be obtained by scaling and translation, while (1.15) can be deduced by construction a class of specific test functions.

In the sequel, we mainly devote to the proof of the sufficiency. In addition, if $a=0$, then (1.12) obviously holds; if $a=1$, we complete the proof in Remark 1.3. So we need only to treat the case $0<a<1$.

4.1 - Sufficiency when $0<a<1,0 \leq \alpha-\sigma \leq 1$

For $p \leq\left(\frac{1}{p}+\frac{\alpha-\sigma-1}{Q}\right)^{-1} \leq p^{*}$, an argument similar to the one in the Remark 1.3, shows that there exists $t(0 \leq t \leq 1)$ such that $\left(\frac{1}{p}+\frac{\alpha-\sigma-1}{Q}\right)^{-1}=\frac{p(Q-t p)}{Q-p}$ and $(\alpha-\sigma)\left(\frac{1}{p}+\frac{\alpha-\sigma-1}{Q}\right)^{-1}=t p$. Hence,

$$
\begin{aligned}
& \int_{G}\left(\frac{|x|}{\rho}\right)^{(\alpha-\sigma)\left(\frac{1}{p}+\frac{\alpha-\sigma-1}{Q}\right)^{-1}} \frac{\left|\rho^{\alpha} u\right|^{\left(\frac{1}{p}+\frac{\alpha-\sigma-1}{Q}\right)^{-1}}}{\rho^{(\alpha-\sigma)\left(\frac{1}{p}+\frac{\alpha-\sigma-1}{Q}\right)^{-1}}} d x d y \\
\leq & C\left(\int_{G}\left|\rho^{\alpha} X u\right|^{p} d x d y\right)^{\frac{Q-t p}{Q-p}} .
\end{aligned}
$$

By (1.11) and (1.13), $\frac{1}{r}=a\left(\frac{1}{p}+\frac{\alpha-\sigma-1}{Q}\right)+\frac{1-a}{q} \leq 1$. For $r>1$, we have 


$$
\begin{aligned}
& \int_{G}\left(\frac{|x|}{\rho}\right)^{(\alpha-\gamma) r} \rho^{\gamma r}|u|^{r} d x d y \\
\leq & \left(\int_{G}\left(\frac{|x|}{\rho}\right)^{(\alpha-\sigma)\left(\frac{1}{p}+\frac{\alpha-\sigma-1}{Q}\right)^{-1}} \frac{\left|\rho^{\alpha} u\right|^{\left(\frac{1}{p}+\frac{\alpha-\sigma-1}{Q}\right)^{-1}}}{\rho^{(\alpha-\sigma)\left(\frac{1}{p}+\frac{\alpha-\sigma-1}{Q}\right)^{-1}}} d x d y\right)^{a\left(\frac{1}{p}+\frac{\alpha-\sigma-1}{Q}\right)} \\
& \cdot\left(\int_{G}\left(\frac{|x|}{\rho}\right)^{(\alpha-\beta) q} \rho^{\beta q}|u|^{q} d x d y\right)^{\frac{1-a}{q}}\left(\int_{G}\left(\frac{|x|}{\rho}\right)^{(\alpha-\gamma) r} \rho^{\gamma r}|u|^{r} d x d y\right)^{\frac{r-1}{r}} \\
\leq & \left(\int_{G}\left|\rho^{\alpha} X u\right|^{p} d x d y\right)^{\frac{a}{p}} \\
& \cdot\left(\int_{G}\left(\frac{|x|}{\rho}\right)^{(\alpha-\beta) q} \rho^{\beta q}|u|^{q} d x d y\right)^{\frac{1-a}{q}}\left(\int_{G}\left(\frac{|x|}{\rho}\right)^{(\alpha-\gamma) r} \rho^{\gamma r}|u|^{r} d x d y\right)^{\frac{r-1}{r}},
\end{aligned}
$$

and hence (1.12) holds. For $r=1$, we argue with the same way.

4.2 - Sufficiency when $0<a<1, \alpha-\sigma>1$

(1.15) tells us $a<1, \frac{1}{p}+\frac{\alpha-1}{Q} \neq \frac{1}{r}+\frac{\gamma}{Q}$. Using the discussion in the end of Section 2, we assume $A^{a} B^{1-a}=1$ and want to prove that

$$
\int_{G}\left(\frac{|x|}{\rho}\right)^{(\alpha-\gamma) r} \rho^{\gamma r}|u|^{r} d x d y
$$

is bounded. In Subsection 4.1, (1.12) has been checked for $\alpha-\sigma=1$ and $\alpha-\sigma=0$. Hence, we conclude that

$$
\int_{G}\left(\frac{|x|}{\rho}\right)^{(\alpha-\delta) s} \rho^{\delta s}|u|^{s} d x d y \leq C, \quad \int_{G}\left(\frac{|x|}{\rho}\right)^{(\alpha-\varepsilon) t} \rho^{\varepsilon t}|u|^{t} d x d y \leq C,
$$

where $\delta, s, \varepsilon$ and $t$ satisfy

$$
\left\{\begin{array}{l}
\delta=b \cdot \alpha+(1-b) \beta, \\
\frac{1}{s}=\frac{b}{p}+\frac{1-b}{q}-\frac{b}{Q}, \\
\varepsilon=d \cdot(\alpha-1)+(1-d) \beta, \\
\frac{1}{t}=\frac{d}{p}+\frac{1-d}{q}
\end{array}\right.
$$


for some choices of $b$ and $d, 0 \leq b, d \leq 1$, and then

$$
\left\{\begin{array}{l}
m+(\alpha-\delta) s>0, \quad \delta s+Q>0 \\
m+(\alpha-\varepsilon) t>0, \quad \varepsilon t+Q>0
\end{array}\right.
$$

Obviously,

$$
\begin{aligned}
& \frac{1}{t}+\frac{\varepsilon}{Q}=d\left(\frac{1}{p}+\frac{\alpha-1}{Q}\right)+(1-d)\left(\frac{1}{q}+\frac{\beta}{Q}\right), \\
& \frac{1}{r}+\frac{\gamma}{Q}=a\left(\frac{1}{p}+\frac{\alpha-1}{Q}\right)+(1-a)\left(\frac{1}{q}+\frac{\beta}{Q}\right), \\
& \frac{1}{s}+\frac{\delta}{Q}=b\left(\frac{1}{p}+\frac{\alpha-1}{Q}\right)+(1-b)\left(\frac{1}{q}+\frac{\beta}{Q}\right) .
\end{aligned}
$$

1) Case $\frac{1}{p}+\frac{\alpha-1}{Q}<\frac{1}{q}+\frac{\beta}{Q}$. Take $b<a<d$ and then

$$
\frac{1}{t}+\frac{\varepsilon}{Q}<\frac{1}{r}+\frac{\gamma}{Q}<\frac{1}{s}+\frac{\delta}{Q}
$$

A direct computation shows that

$$
\begin{aligned}
& \frac{1}{r}-\frac{1}{s}=(a-b)\left(\frac{1}{p}-\frac{1}{Q}-\frac{1}{q}\right)+\frac{a(\alpha-\sigma)}{Q}, \\
& \frac{1}{r}-\frac{1}{t}=(a-d)\left(\frac{1}{p}-\frac{1}{q}\right)+\frac{a(\alpha-\sigma-1)}{Q}, \\
& \left(\frac{1}{r}+\frac{\alpha-\gamma}{m}\right)-\left(\frac{1}{t}+\frac{\alpha-\varepsilon}{m}\right) \\
& =(a-d)\left(\frac{1}{p}-\frac{1}{q}+\frac{1}{m}(\beta+1-\alpha)\right)+a(\alpha-\sigma-1)\left(\frac{1}{Q}+\frac{1}{m}\right), \\
& \left(\frac{1}{r}+\frac{\alpha-\gamma}{m}\right)-\left(\frac{1}{s}+\frac{\alpha-\delta}{m}\right) \\
& =(a-b)\left(\frac{1}{p}-\frac{1}{q}-\frac{1}{Q}+\frac{1}{m}(\beta-\alpha)\right)+a(\alpha-\sigma)\left(\frac{1}{Q}+\frac{1}{m}\right),
\end{aligned}
$$

Since $a>0, \alpha-\sigma>1$, we see

$$
\begin{aligned}
& 0<\frac{a(\alpha-\sigma-1)}{Q}<\frac{a(\alpha-\sigma)}{Q}, \\
& 0<a(\alpha-\sigma-1)\left(\frac{1}{Q}+\frac{1}{m}\right)<a(\alpha-\sigma)\left(\frac{1}{Q}+\frac{1}{m}\right)
\end{aligned}
$$


and for sufficiently small $|d-a|$ and $|b-a|$,

$$
\left\{\begin{array}{l}
\frac{1}{r}>\frac{1}{s}, \quad \frac{1}{r}>\frac{1}{t} \\
\frac{1}{r}+\frac{\alpha-\gamma}{m}>\frac{1}{t}+\frac{\alpha-\varepsilon}{m} \\
\frac{1}{r}+\frac{\alpha-\gamma}{m}>\frac{1}{s}+\frac{\alpha-\delta}{m}
\end{array}\right.
$$

Combining (4.5) and (4.6), one gets

$$
\begin{cases}m+(\varepsilon-\gamma) \frac{t r}{t-r}>0, & (\gamma-\varepsilon) \frac{t r}{t-r}+Q>0, \\ m+(\delta-\gamma) \frac{s r}{s-r}>0, & (\gamma-\delta) \frac{s r}{s-r}+Q<0 .\end{cases}
$$

By Hölder inequality it yields

$$
\begin{aligned}
& \left(\int_{G}\left(\frac{|x|}{\rho}\right)^{(\alpha-\gamma) r} \rho^{\gamma r} \Phi|u|^{r} d x d y\right)^{\frac{1}{r}} \\
\leq & C\left(\int_{G}\left(\frac{|x|}{\rho}\right)^{(\alpha-\varepsilon) t} \rho^{\varepsilon t}|u|^{t} d x d y\right)^{\frac{1}{t}}
\end{aligned}
$$

and

$$
\begin{aligned}
& \left(\int_{G}\left(\frac{|x|}{\rho}\right)^{(\alpha-\gamma) r} \rho^{\gamma r}(1-\Phi)|u|^{r} d x d y\right)^{\frac{1}{r}} \\
\leq & \left(\int_{G}\left(\frac{|x|}{\rho}\right)^{(\alpha-\delta) s} \rho^{\delta s}|u|^{s} d x d y\right)^{\frac{1}{s}},
\end{aligned}
$$

where $\Phi$ is defined in (2.2). Combining (4.2), (4.8) and (4.9), we conclude that (4.1) holds.

2) Case $\frac{1}{p}+\frac{\lambda-1}{Q}>\frac{1}{q}+\frac{\beta}{n}$. Take $d<a<b$ such that $|d-a|$ and $|b-a|$ are sufficiently small. Then (4.3)-(4.7) hold. This implies that (4.2), (4.8) and (4.9) are true. So (4.1) holds. 
Acknowledgments. The authors would like to express the gratitude to the referees for their valuable mentions and suggestions.

\section{REFERENCES}

[1] M. Badiale - G. TARANTEllo, A Sobolev-Hardy inequality with applications to a nonlinear elliptic equation arising in astrophysics, Arch. Rational Mech. Anal., 163 (2002), pp. 259-293.

[2] Z.M. Balogh - J.T. Tyson, Polar coordinates in Carnot groups, Math. Z. 241 (2002), pp. 697-730.

[3] G. Barbatis - S. Filippas - A. Tertikas, A unified approach to improved $L^{p}$ Hardy inequalities with best constants, Trans. Amer. Math. Soc., 356(6) (2004), pp. 2169-2196.

[4] L. CAfFARElli - R. KoHN - L. Nirenberg, First order interpolation inequalities with weights. Compositio Math., 53 (1984), pp. 259-275.

[5] F. CATRINA - Z.Q. WANG, On the Caffarelli-Kohn-Nirenberg inequalities: sharp constants, existence (and nonexistence), and symmetry of extremal functions, Comm. Pure and Applied Math., 54 (2001), pp. 229-258.

[6] N. Chaudhuri, On Hardy-Sobolev inequality and its application to certain singular problems, PH.D Thesis, Indian Institute of Science, 2001.

[7] J.L. CheRN - C.S. LIN, Minimizers of Caffarelli-Kohn-Nirenberg inequalities with the singularity on the boundary. Arch. Rational Mech. Anal., 197 (2010), pp. 401-432.

[8] G.B. Folland - E.M. Stein, Hardy spaces on homogeneous groups, Princeton Univ. Press, Princeton 1980.

[9] J.P. García Azorero - I. Peral Alonso, Hardy inequalities and some critical elliptic and parabolic problems, J. Differential equations, 144 (1998), pp. 441-476.

[10] N. GAROFAlO - D. VASSILEV, Regularity near the characteristic set in the nonlinear Dirichlet problem and conformal geometry of sub-Laplacians on Carnot groups, Math. Ann., 318 (2000), pp. 453-516.

[11] N. Garofalo - D. Vassilev, Symmetry properties of positive entire solutions of Yamabe-type equations on groups of Heisenberg type, Duke Math. J., 106(3) (2001), pp. 411-448.

[12] N. Ghoussoub - C. Yuan, Multiple solutions for quasilinear PDE involving the critical Sobolev exponent, Trans. Amer. Math. Soc., 352 (2000), pp. 57035743.

[13] C.E. Gutierrez - R.L. Wheeden, Sobolev interpolation inequalities with weights, Trans. Amer. Math. Soc., 323 (1991), pp. 263-281.

[14] J.Q. HAN - P.C. NIU, Polar coordinates on H-type groups and applications, Vietnam J. Math., 34(3) (2006), pp. 307-316.

[15] Y.Z. Han - P.C. NiU, Hardy-Sobolev type inequalities on the H-type group, Manuscripta Math., 118 (2005), pp. 235-252.

[16] Y.Z. HAN - P.C. NIU - S.T. ZHANG, On first order interpolation inequalities with weights on the Heisenberg group, Acta Math. Sinica (Engl. Ser.), 27(12) (2011), pp. 2493-2506. 
[17] Y.Z. HAN - S.T. ZHANG - J.B. Dou, On first order interpolation inequalities with weights on the H-type group, Bull. Braz. Math. Soc., (N. S.) 42(2) (2011), pp. 185-202.

[18] A. KaPLAN, Fundamental solutions for a class of hypoelliptic PDE generated by composition of quadratic forms, Trans. Amer. Math. Soc., 258 (1980), pp. 147-153.

[19] T. Kusano - J. Jaroš - N. Yoshida, A Picone-type identity and Sturmian comparison and oscillation theorem for a class of half-linear partial differential equations of second order, Nonlinear Anal., 40 (2000), pp. 381-395.

[20] C. LiN - Z.Q. WANG, Symmetry of extremal functions for the Caffarelli-KohnNirenberg inequalities, Proc. Amer. Math. Soc., 132 (2004), pp. 1685-1691.

[21] C.S. Lin, Interpolation inequalities with weights. Comm. in Partial Differential Equations, 11(14) (1986), pp. 1515-1538.

[22] G.Z. Lu, Local and global interpolation inequalities on the Folland-Stein Sobolev spaces and polynomials on stratified groups, Math. Res. Lett., 4(6) (1997), pp. 777-790.

[23] G.Z. Lu, Polynomials, higher order Sobolev extension theorems and interpolation inequalities on weighted Folland-Stein spaces on stratified groups, Acta Math. Sinica (Engl. Ser.), 16(3) (2000), pp. 405-444.

[24] D.N. Vassilev, Yamabe equations on Carnot groups, PH.D Thesis, Purdue University, December 2000.

Manoscritto pervenuto in redazione il 17 Gennaio 2013. 